\title{
Ecología del comportamiento en aulas para niños disminuidos de preescolar *
}

\section{Ann K. Rogers-Warren **}

Universidad de Kansas

Traducción: Genís Sánchez

Si bien durante los últimos quince años se ha dado un gran avance en el desarrollo de planes de estudio y programas de servicios generales para niños disminuidos, la tecnología para llevar a cabo una intervención eficaz en este campo todavía es incompleta. Un aspecto de la planificación que hasta el momento no se ha explorado en profundidad es un diseño de las aulas y de los lugares destinados al tratamiento que facilite la enseñanza y la prestación de los servicios. Los servicios de salud federales, estatales y locales proporcionan unas directrices mínimas para el diseño de las aulas mediante la regulación de su tamaño, sus condiciones sanitarias y la cantidad de personal. Estas normas no ofrecen directrices para la creación de ambientes que den apoyo a los niños disminuidos y a sus maestros. La planificación ambiental posee un rico acervo de propuestas basadas en el sentido común, que ha sido compartido formalmente (en libros de texto) e informalmente por generaciones de maestros. A pesar de que muchas de estas propuestas son excelentes, pocas de ellas se basan en datos concretos.

No se ha llevado a cabo ningún estudio experimental para investigar el efecto de las variables del escenario en el comportamiento de los niños disminuidos. Hay varios estudios descriptivos de corte ecológico que evalúan informalmente ( $\sin$ utilizar diseños de investigación específicos) el efecto del marco ambiental en el juego y el aprendizaje de niños normales (por ejemplo, Gump, 1969). Unos pocos estudios de ecologia del comportamiento han examinado, mediante diseños de grupo o de sujeto único, los efectos de determinadas condiciones ambientales no sociales en el comportamiento de los niños (por ejemplo, Quilitch y Risley, 1973). Además, en publicaciones especializadas en el análisis del comportamiento (por ejemplo, Journal of Applied Bebavior Analysis, 1968-1981), se encuentran muchas evaluaciones experimentales de los efectos de condiciones sociales en el comportamiento de niños normales y disminuidos. Sólo un número muy pequeño de estudios han incluido niños disminuidos menores de cinco años. Dada la ausencia de literatura centrada en la planificación ambiental, sólo pueden obtenerse recomendaciones sobre el tema rebuscando en los estudios ya existentes, los cuales abarcan un amplio abanico de comportamientos en sujetos con distintas edades y niveles de aptitud.

\section{EL ENTORNO \\ DEL APRENDIZAJE}

El aula consta tanto de componentes físicos como sociales. Los componentes

* «Behavioral ecology in classrooms for young, handicapped children» Topics in Early Childbood Special Education, 1982, 2:I, 21-32. Reproducido con autorización. (9) de esta traducción, Infantia y Aprendizaje; 1985.

** Directión de la autora: Bureau of Child Research, Department of Human Development, University of Kansas, Lawrence, Kansas 66045, USA. 
mom físicos incluyen, a grandes rasgos, el es-. pacio real del aula, la disposición de las áreas de actividad dentro de este espacio, los muebles y las instalaciones fijas, los materiales empleados en el juego y el trabajo (no considerados aquí como materiales especificos del plan de estudios), las actividades del programa y su secuencia, la cantidad de personal, la cantidad de niños, disminuidos y no disminuidos, y los grupos formados por los niños y el personal a su cargo. Los componentes sociales estriban en los comportamientos de los adultos y de los niños en el marco educativo.

Este artículo se centra, principalmente, en los componentes físicos del aula; sin embargo, es imposible separar los efectos de estos componentes de los efectos de los componentes sociales. En un ambiente dado, la gente se comporta de una manera determinada y este comportamiento también forma parte del escenario. Un aula es un sistema dinámico. Los cambios en el aula afectarán tanto a los niños como al personal y también el comportamiento del personal repercutirá en el comportamiento de los niños y resulta de gran utilidad examinar por separado estos dos tipos de cambios en los estudios experimentales. Puede que al profesional no le importe saber cómo ocurren los cambios que llevan a resultados positivos, siempre y cuando éstos se den. En ciertos casos, pueden utilizarse variables sociales (como la atención del maestro) para establecer el contacto de un niño con el marco educativo o para desarrollar nuevas destrezas. Por estas razones, se propone un enfoque algo amplio de las condiciones ambientales, enfoque que no menosprecia la importancia que tiene el diseño del entorno físico; simplemente indica que los componentes sociales y físicos del marco educativo interactúan entre si y. que deberían ser examinados conjuntamente.

El entorno ideal de un aula es aquel que favorece los comportamientos y destrezas apropiados para el grupo de niños inscritos en ella. De acuerdo con este supuesto, en este artículo se describen disposiciones ambientales que favorecen comportamientos que se consideran importantes en la intervención con niños disminuidos del preescolar. Concretamente se describen procedimientos para:

- fomentar la interacción social;
- facilitar el aprendizaje del lenguaje y la comunicación;

- diseñar marcos de instrucción que estimulen el aprendizaje;

- dirigir el comportamiento;

- desarrollar la independencia, posibilitar los intercambios, y

- facilitar el comportamiento del personal.

También se abordarán otros dos temas: la evaluación de las disposiciones ambientales y si satisfacen las necesidades especiales de niños físicamente disminuidos.

\section{FOMENTO DE LA INTERACCION SOCIAL}

Los ambientes de preescolar deberían estimular la interacción social entre los niños y sus compañeros, y darles oportunidades para aprender nuevas destrezas sociales. Hay varias condiciones relativas al aula que pueden facilitar el desarrollo social: incluir niños que puedan actuar como compañeros de juego y como modelo de comportamientos deseados; incluir materiales que estimulen la interacción social, y disponer el personal y las actividades de modo que permitan el contacto entre los niños. Algunas de estas condiciones son ya habituales en las clases integradas; sin embargo, antes de maximizar el impacto de estas condiciones vamos a examinarlas a la luz de su importancia para fomentar el desarrollo social de los niños disminuidos.

\section{Niños disminuidos y no disminuidos: jugar $y$ aprender juntos}

Para que los niños disminuidos se beneficien de la presencia de compañeros normales en el aula, deben entrar en. contacto con ellos, sea indirectamente (viéndoles llevar a cabo comportamientos adecuados) o directamente (mediante intercambios sociales verbales o no verbales). Cuando los niños disminuidos juegan con compañeros de clase no disminuidos, en los primeros se da un aumento del juego paralelo y cooperativo (RogersWarren, Ruggles, Peterson \& Cooper, en prensa). Sin embargo, frecuentemente se da ségregación en vez de integración (Peterson \& Haralick, 1977), y la imitación espontánea de los modelos ofrecidos 
por los compañeros es muy rara (Guralnick, 1978; Sullivan, 1977). Reunir niños disminuidos y no disminuidos en la misma clase puede ser un método necesario, aunque no enteramente suficiente, de incrementar las competencias en la interacción social. Para obtener los máximos efectos beneficiosos de estas situaciones, pueden ser necesarias otras intervenciones sociales. Por ejemplo, podría ser útil que el maestro emparejase a niños con diferentes competencias mediante sugerencias y elogios, disponiendo de antemano los asientos a ocupar, y fijando unas reglas que requieran traer a un amigo para participar en actividades atractivas. También puede ser necesario un adiestramiento específico para imitar a compañeros o interactuar con ellos.

\section{Materiales que estimulan la interacción social}

Algunos materiales estimulan la interacción social más que otros. En niños de preescolar y de primera enseñanza, los vagones, bloques huecos, materiales de juego dramático y los juegos que requieren dos participantes, fomentan la conversación y la actividad conjunta (Quilitch y Risley, 1973; Shure, 1963; Van Alatyne, 1932).

Los niños disminuidos de preescolar muestran pautas similares de juego social, interactúan más con los compañeros durante el juego con bloques $y$ en juegos manipulativos en el suelo, que en actividades artísticas y «de mesa» (Peterson y Haralick, 1977). En todas las actividades, los niños disminuidos están más aislados socialmente (sin participar en ellas), que los niños no disminuidos. Los niños disminuidos también muestran niveles de juego menos sofisticados que sus compañeros normales en las mismas áreas (Rogers-Warren et al., en prensa). La presencia de materiales $y$ actividades sociales puede afectar a los niños disminuidos, aunque puede no estimular la interacción social en estos niños con la misma intensidad con que lo hace en niños normales. Por ejemplo, las actividades de juego dramático que dan pie a conversaciones y a juegos imaginativos pueden tener un efecto menor en niños con competencias de comunicación limitadas.

\section{Número de maestros}

Normalmente se propone una mayor proporción personal-niños ( $\mathrm{i}: 4$ contra I: 10$)$ en programas para niños disminuidos. Hay pocos estudios que hayan documentado los efectos de la proporción maestros-niños en las interacciones sociales de niños disminuidos de preescolar. Sin embargo, los niños de preescolar normales interactúan con sus compañeros con menos frecuencia cuando las proporciones son mayores ( $1: 3,5$ contra $1: 7$; O'Connor, I 975). Un mayor número de adultos tiende a incrementar los tiempos de transición y a disminuir la participación de los niños en las actividades, especialmente en el caso de niños muy pequeños (Stodolosky, 1974).

La presencia de adultos puede hacer algo más que desempeñar un papel mediador con niños disminuidos Los adultos atraen a los niños a áreas de actividad y mantienen su interacción con materiales (Hursh, 1973). Cuando las aptitudes para interactuar con compañeros son muy limitadas (como ocurre con niños recluidos en instituciones), los adultos representan la mayoría de oportunidades para la conversación y la interacción social (Berkson y Landesman-Dwyer, I977). Hace falta llevar a cabo más investigaciones sobre los efectos de la proporción personal-niños en la interacción social.

\section{FACILITACION DEL APRENDIZAJE DEL LENGUAJE Y DE LA COMUNICACION}

Aunque hay muchas referencias sobre la corrección de deficiencias del lenguaje, se han realizado pocos estudios sobre las condiciones del aula que estimulan su aprendizaje. Como norma general, las recomendaciones para facilitar la interacción social se aplican también al aprendizaje del lenguaje. Cuando los niños entran en contacto mutuo tienen la oportunidad de usar y aprender el lenguaje. Además, las aulas deberian apoyar la generalización de las competencias comunicativas recién aprendidas, y proporcionar oportunidades de aprender otras nuevas. 
Importancia de los materiales y de la participación de los niños

El primer paso a dar para estimular el uso del lenguaje en preescolar es hacer que el niño interactúe con materiales (Hart y Risley, 1975). Si el niño interactúa con materiales y se ve reforzado por estos contactos, habrá oportunidades de enseñar nuevas aptitudes y apoyar el uso del lenguaje. Disponer los materiales de modo que algunos de los más atractivos sean visibles, pero no inmediatamente accesibles, hará que aumente el contacto del niño con los adultos o compañeros que distribuyan estos materiales. La utilización de materiales en situaciones de grupo similares a las empleadas en la enseñanza individualizada, puede estimular la generalización de vocablos recién aprendidos, especialmente en niños disminuidos (Warren y Rogers-Warren, 1980); sin embargo, inicialmente puede ser necesario que el maestro haga preguntas o realice indicaciones dando pistas para las respuestas a estos materiales. Los materiales nuevos pueden provocar preguntas en niños con competencias más sofisticadas (Warren, Baxter, Anderson, Marshall y Baer, 198r).

\section{Rutinas}

Las rutinas proporcionan a los niños modelos de verbalizaciones adecuadas y crean situaciones en las que estas verbalizaciones son provocadas por señales no verbales consistentes. A medida que aumentan las aptitudes verbales de los niños, éstos completan los componentes verbales de la interacción y anticipan los siguientes pasos de la rutina (Moerk, 1977). Halle, Marshall y Spradlin (1979) mostraron una manera de estimular el uso espontáneo del lenguaje en adolescentes retrasados mediante rutinas. Cuando los estudiantes se aproximaban al mostrador del autoservicio para recoger sus bandejas de comida, el encargado aguantaba la bandeja y no la soltaba hasta que decian «la bandeja, por favor", respuesta que ya había sido previamente moldeada en el contexto de esta rutina.

En un segundo estudio, Halle (1981) demostró que los maestros de niños disminuidos de preescolar podian interrumpir rutinas establecidas (para comer algo, salir de la clase, ir al lavabo, etc.) y, al retrasar el siguiente paso de la rutina, incitar a los niños a pedirla verbalmente. Esta estrategia para desarrollar peticiones espontáneas depende igualmente de la estructura del entorno (rutinas, acceso limitado a algunos materiales o acontecimientos reforzadores) y de la mediación del maestro (retrasar el paso siguiente y ya familiar de la rutina, como incitación al lenguaje).

\section{Comunicación con los compañeros}

La importancia que tiene la presencia de compañeros competentes en las clases de niños disminuidos es puesta de relieve en un estudio de Paul, Rogers-Warren y Spradlin (1978). Unos niños de preescolar que presentaban retraso en el lenguaje hablaban más en grupos compuestos por un niño con retraso en el lenguaje y dos niños normales, que en grupos compuestos por tres niños retrasados. Después de haber practicado con niños normales, algunos niños disminuidos incrementaron su habla en presencia de compañeros retrasados. Sin embargo, se observó que unos niños con retraso en el lenguaje hablaban menos en un aula especial de integración que en las aulas de preescolar y guarderías a las que normalmente asistían (Paul y McQuarter, 1979). Así pues, la presencia de compañeros competentes no garantiza la comunicación, aunque sí aumenta su probabilidad.

\section{Comunicación con los maestros}

La presencia de un maestro también hace que los niños que presentan retrasos en el lenguaje hablen más (Warren y Rogers-Warren, 1980); sin embargo; una interacción verbal muy alta con los maestros limita la interacción verbal de los niños con sus compañeros (RogersWarren y Warren, 1980). El número y la disponibilidad de los maestros debería determinarse en base a los objetivos marcados respecto a la comunicación de los niños en el aula. La construcción de competencias básicas para la comunicación y el fomento de verbalizaciones frecuentes requieren un entorno altamente responsivo para cuya obtención es necesaria la presencia atenta de varios maestros. Para estimular las interacciones ver- 
bales con los compañeros puede ser necesario limitar el número de maestros o la frecuencia de las interacciones con otros niños iniciadas por ellos.

\section{DISEÑO DE ESCENARIOS DE INSTRUCCION QUE ESTIMULEN EL APRENDIZAJE}

Los escenarios especiales de instrucción se diseñan normalmente para conseguir una enseñanza más individual y con menos distracciones. Sin embargo, el uso de ambientes restringidos, como cubiculos dentro del aula, ha demostrado tener pocos efectos positivos en el aprendizaje infantil (véase Cruickshank, Bentzen, Ratzeburg y Tannehauser, 196r; Haring y Phillips, 1967; Shores y Haubrich, 1969). La generalización de competencias recièn adquiridas, la preparación de los estudiantes para escenarios educativos normales y las limitaciones en la cantidad de personal hacen necesario disminuir la cantidad de enseñanza impartida uno/uno.

\section{Tamaño de los grupos}

Varios estudios han demostrado que los niños retrasados y autistas aprenden igual o mejor en grupos pequeños, donde tienen la oportunidad de observar a compañeros, que en situaciones de enseñanza uno/uno (véase Biberdorf y Pear, 1977; Favell, Favell y McGimsey, 1978; Frankel y Graham, 1976). Aunque no se han llevado a cabo investigaciones sobre el aprendizaje de niños disminuidos en grupos mayores (más de diez niños), los estudios realizados por Barker y Gump (1965) y Dawes (1934) indican que la participación de los niños normales disminuye a medida que aumenta el tamaño del grupo.

\section{Densidad y distribución espacial}

Muchas investigaciones se han dedicado a examinar los efectos de la densidad en ambientes normales de preescolar (véase Loo, I972; McGrew, 1970). Los comportamientos disruptivos aumentan con la masificación, pero normalmente los maestros minimizan estos efectos actuando de forma más directiva (Fagot, 1977). Si bien no se han realizado estudios sobre los efectos de la masificación en niños disminuidos, Krantz y Risley (1977) indicaron que si durante un período de instrucción se distribuían los asientos de niños de preescolar alrededor del maestro, de forma que quedara espacio entre cada asiento, aumentaba la atención hacia la tarea y el maestro. Siruar a los niños juntos sobre una alfombra de grandes proporciones disminuia la atención e incrementaba las perturbaciones. Sin embargo, bastaba una actitud positiva hacia el comportamiento adecuado para restituir niveles elevados de atención durante la condición de masificación.

\section{Programas de actividades}

Los programas de actividades coherentes estimulan la atención hacia las tareas. En el contexto de un programa coherente, una secuencia que gradualmente disminuya el nivel de actividad (por ejemplo, desde un juego muy activo hasta una instrucción en grupo) puede limitar las interrupciones y aumentar la atención de los niños (Krantz y Risley, 1977). Los cambios de escenario (pasar del exterior al interior) también pueden fomentar la atención durante los períodos de instrucción (Hawn, Holt y Holmberg, 1973). Realizar un programa de actividades superpuestas es una manera excelente de limitar las interrupciones y de aumentar la participación de los niños (Doke y Risley, 1972; LeLaurin y Risley, 1972). La composición de actividades elimina las transiciones en grupo que contribuyen a interrumpir el comportamiento.

Las actividades programadas de forma escalonada ofrecen a los niños con distintas capacidades de atención continuas oportunidades de participación y pueden constituir un diseño especialmente útil para las clases integradas.

\section{CONTROL DEL COMPORTAMIENTO}

Los niños disminuidos plantean con frecuencia problemas específicos para el control de su comportamiento. Aunque ya contamos con una tecnología bien desarrollada para reducir los comportamientos inadecuados y aumentar las competencias positivas, una cuidadosa estructuración del aula puede facilitar la aplica- 
ción de los procedimientos de control del comportamiento $y$, en algunos casos, hacer innecesaria su aplicación.

\section{Prevención del comportamiento disruptivo}

Las perturbaciones se dan con más frecuencia cuando los niños esperan que empiecen las actividades, pasan de un área a.otra o mantienen un contacto muy estrecho entre sí (Fowler, en prensa). Las actividades simultáneas o superpuestas reducen el tiempo de espera de los niños. Si se deja que los niños pasen a la siguiente actividad cuando están listos para hacerlo, su participación aumenta y disminuyen las interrupciones (Doke y Risley, 1972). Asignar maestros a actividades en vez de a determinados niños favorece el control de su comportamiento; este control también puede reducir las perturbaciones (LeLaurin y Risley, 1972). Impidiendo que los niños se amontonen en espacios reducidos y programando los momentos de actividad y reposo en períodos adyacentes, también se limitan las interrupciones (Krantz y Risley, 1977). Emparejar a los niños para que realicen actividades les permite contemplar modelos de comportamiento adecuados y las reacciones del maestro a estos comportamientos, con lo que se puede reducir el comportamiento problemático sin intervenir directamente (Peck, Cooke y Apolloni, 1981). El establecimiento de límites claros para cada actividad mediante la distribución de los muebles también ayuda a los niños a aprender dónde se espera que se lleven a cabo ciertos comportamientos (Fowler, en prensa). Si se escoge cuidadosamente la localización de las actividades que requieren espacio, de manera que éstas no bloqueen el paso o el acceso a otras áreas, es probable que se reduzca la circulación de un lado a otro, asi como las perturbaciones (Kritchevsky, Prescott y Walling, 1977).

\section{Modificación del comportamiento disruptivo}

Cuando hay que aplicar un procedimiento específico para limitar el comportamiento disruptivo, la estructuración ambiental puede maximizar sus efectos e, idealmente, limitar la frecuencia de su uso. Por ejemplo, para que los procedimientos de descanso sean efectivos, el escenario debe ser atractivo y reforzador para el niño, debe haber frecuentes oportunidades de participar positivamente con materiales, maestros y compañeros. Los procedimientos de castigo muy breves 0 suaves (como sentarse y observar; Porterfield, Herbert-Jackson y Risley, 1976) serán muy eficaces en cscenarios atractivos. Los descansos son más eficaces cuando el área de descanso está lejos de vistas y sonidos agradables y fuera de áreas transitadas. Tener un asiento fijo para el descanso incrementará su eficacia, de la misma manera que el empleo de áreas claramente delimitadas para las diversas actividades mejorará el comportamiento adecuado en estas áreas.

\section{CONSTRUIR}

\section{LA INDEPENDENCIA \\ Y FACILITAR LA TRANSICION}

En la literatura educativa destaca la ausencia de investigaciones sistemáticas en torno a las condiciones ambientales que fomentan la adquisición de autocontrol $\mathrm{e}$ independencia en las aulas. Recientemente, Fowler (en prensa) y sus colegas han empezado a investigar algunas de las condiciones ambientales que facilitan escenarios más típicos de la escuela pública, aunque la necesidad de investigar en este campo continúa siendo muy grande.

En vez de estar basadas en datos obtenidos a partir de investigaciones concretas, las propuestas que vienen a continuación se basan en directrices de diseño ambiental para disminuidos (Cary, 1978), en análisis limitados de ambientes para niños normales de preescolar (Montes y Risley, 1975), y en algunos datos obtenidos en ambientes institucionales para residentes gravemente disminuidos (Sandhu y Hendricks-Jansen, 1976; Zukotynski y Ogolsky, 1981).

\section{Desarrollo de la independencia}

Muchos planes de estudio para niños disminuidos desarrollan la independencia mediante la instrucción en técnicas de esfuerzo personal y lenguaje. El ambiente del aula puede facilitar la consecución de estas metas, proporcionando oportunidades para que los niños dirijan su propio 
comportamiento y se encarguen de parte de su material.

Muchos de los aspectos del acondicionamiento de las aulas discutidos anteriormente estimulan el desarrollo de la independencia. Las aulas deberían permitir al niño el acceso a aquello que se necesita con frecuencia: el baño, las fuentes para beber y los materiales de juego. Aunque muchos niños disminuidos no pueden encargarse por sí solos de los materiales o de las rutinas de cuidado personal, incluso el más pequeño de los esfuerzos puede contribuir al desarrollo de la independencia. Cuando los cuartos de baño están justamente al lado de las clases y están equipadas con barras para aguantarse y taburetes con peldaños, los niños con aptitudes físicas moderadas pueden llevar a cabo por lo menos una parte de las actividades de aseo y limpieza de manos, con un grado minimo de supervisión. Incluso los niños en silla de ruedas y con minima movilidad pueden seleccionar materiales de estanterías abiertas que estén a una altura adecuada.

La adopción de simples códigos de color para los materiales y las áreas para guardarlos ayudará a los niños pequeños $o$ incapacitados a encontrar y guardar los materiales. Montes y Risley (1975) llegaron a la conclusión de que, en realidad, los niños pasan más tiempo jugando con juguetes manipulativos cuando éstos se guardan en estanterias abiertas, que cuando se guardan en cajas en las que el niño debe rebuscar para encontrar el objeto deseado. Para estimular el uso de materiales por parte de los niños, los objetos deben guardarse de manera que. sean fácilmente accesibles.

Si se permite a un niño tener algunos materiales (por ejemplo, lápices de colores o libretas) en un cajón o un armario pequeño se preparará al niño para el sistema de auto-administración de materiales empleado en la escuela pública. Proporcionar a los niños la oportunidad de servir zumos, distribuir servilletas, preparar cosas para comer, elegir los lugares para sentarse o seleccionar un instrumento musical, son buenos métodos para permitirles controlar pequeños aspectos de su entorno. (En este caso, el ajuste ambiental de apoyo es la ausencia de un ambiente predeterminado.) Una rutina diaria coherente también facilitará el funcionamiento independiente del niño $y$ sólo requerirá un minimo de instrucciones.

El establecimiento de interacciones con el entorno puede ser una meta preliminar a establecer en el desarrollo de la independencia en niños disminuidos. Se ha visto que el empleo de espejos, objetos móviles e interruptores fácilmente activables que controlen luces, música, aire y diapositivas de personas familiares, aumentan la interacción con el entorno de niños y adolescentes subnormales profundos residentes en instituciones (Sandhu y Hendricks-Jansen, 1976; Zukotynski y Ogolsky, 1981). Entre los rasgos ambientales más necesarios se encuentran la estructuración del marco ambiental para que los niños disminuidos tengan objetos atractivos a su alcance, y el diseño de nuevos sistemas que permitan a estos niños controlar incluso aspectos pequeños de sus entornos cotidianos.

\section{Facilitar la transición}

Las diferencias entre los centros de preescolar, los jardines de infancia públicos y las aulas de enseñanza primaria pueden presentar dificultades para los niños disminuidos que realizan la transición de un escenario a otro. Muchas de las condiciones propuestas como medios de apoyo al comportamiento de los niños no se darán en las aulas de una escuela pública, que tiene más niños y menos maestros. Se puede facilitar una transición fluida al nuevo entorno alterando algunas estructuras ambientales durante los últimos meses de preescolar, para ayudar a que los niños aprendan los comportamientos que les exigirá el nuevo entorno (Fowler, en prensa).

Normalmente, las aulas para niños mayores tendrán pupitres en vez de mesas, habrá menos adultos y menos atención individualizada y se darán periodos más largos para cada actividad y normas más especificas respecto a hablar alto en grupo y durante el trabajo. Variar el ambiente de preescolar para que los niños practiquen el comportamiento adecuado en una gama de ambientes, les ayudará a mantener estos comportamientos en los nuevos marcos ambientales. 


\section{FACILITAR}

\section{EL COMPORTAMIENTO DEL PERSONAL}

Las aulas también constituyen marcos para el comportamiento de los adultos. Para facilitar el comportamiento deseable en los niños, el entorno debe estimular el comportamiento deseable en el personal. Hay relativamente pocas investigaciones que hayan examinado el apoyo dado al personal mediante el diseño de marcos educativos para niños disminuidos. Los trabajos de Risley y sus colegas (ver Herbert-Jackson, O'Brien, Porterfield y Risley, 1977; Twardosz, Cataldo y Risley, 1974) proporcionan la mayoría de los datos básicos y las directrices más claras para estructurar ambientes que estimulen el comportamiento deseable en el personal.

Los ambientes abiertos, caracterizados por divisiones de escasa altura que separan las áreas de actividad, facilitan la supervisión de los niños por parte de los maestros sin interferir con sus actividades (Twardosz et al, 1974). Estas estructuraciones también facilitan la comunicación entre el personal, y les permiten ayudarse mutuamente cuando sea necesario y anticipar las necesidades de los niños a medida que se trasladan de una zona a otra.

La asignación de maestros a zonas o actividades específicas constituye un diseño más eficaz que la asignación a grupos pequeños de niños (LeLaurin y Risley, I 972). En este tipo de estructuración del aula, los maestros sólo se responsabilizan de los niños que entran en su área particular. En este diseño, los niños tienen libertad para pasar de una zona a otra en cuanto completan sus actividades, con lo que la espera es breve o nula, y los maestros emplean más tiempo en enseñar que en dirigir las transiciones. Si se divide el aula en zonas funcionales con todos los materiales necesarios a mano, se reducirán las ausencias del maestro (Herbert-Jackson et al., 1977). Un medio eficaz de mantener informado al personal y supervisar sus actividades es el consistente en colocar en un lugar bien visible los programas de actividad diaria y las responsabilidades de cada miembro (LeLaurin, 1973). La inclusión de objetivos de comunicación y comportamiento para cada niño, así como de indicaciones para procedimientos de deasarrollo de compor-

\section{EVALUACION DE DISEÑOS AMBIENTALES}

Hay muchas maneras de evaluar el respaldo ofrecido por el entorno al comportamiento de los niños y del personal a su. cargo. En cualquier sistema de observación, los comportamientos críticos a seguir en los niños son la ocupación (uso de materiales) y el comportamiento inapropiado. Unos niveles altos de ocupación adecuada indican que el niño se siente reforzado por el entorno (Hart y Risley, 1975). Las medidas de conducta inadecuada son un útil indice del ajuste de un niño al marco ambiental (CardinSmith y Fowler, en prensa; Walker y Hops, 1976). Entre las conductas a observar en el personal se incluyen la presencia, el compromiso con los niños y otras actividades no educativas (por ejemplo, tareas de mantenimiento, interacción con el resto de personal).

Puede usarse el Sistema de Comprobación PLA [Planned Activities Check (Control de Actividades Planificadas), Risley y Cataldo, 1974]para comprobar el comportamiento de los niños a intervalos determinados (por ejemplo, cada seis minutos durante todo el día). Para poner en práctica este sistema, deben especificarse las conductas apropiadas y no apropiadas para cada uno de los períodos diarios de actividad. En cada uno de los intervalos prefijados, se cuenta el número de niños presentes en el área de actividad que participan adecuadamente. Se pueden sumar los datos de cada actividad para cada día y mostrar un porcentaje de las actividades apropiadas como medida de la eficacia ambiental.

Con un programa de exploración (Rogers-Warren, 1975) se obtienen resultados similares. En el procedimiento de exploración, un observador mira sucesivamente a cada niño y a cada miembro del personal (normalmente en un orden determinado) durante tres segundos, y registra el comportamiento de cada individuo observado antes de pasar a observar el siguiente. Los códigos de exploración pueden ser sencillos o complejos según el número y tipo de conductas observadas. Por ejemplo, se pueden definir y observar 
niveles de juego cooperativo o el uso de diversos tipos de material. Los códigos de exploración pueden usarse para determinar el comportamiento de los niños y del personal, de unos niños determinados o solamente del personal. La amplitud de los intervalos y la frecuencia de la exploración, varian de acuerdo con la cantidad y variedad de los comportamientos a observar. Se pueden utilizar códigos de exploración para observar actividades en diversas áreas de forma simultánea o secuencial.

La evaluación del entorno no siempre requiere este tipo de observaciones a intervalos. Muchas variables ambientales permanecen constantes durante todo un período de actividad. Una manera directa de registrar las variables ambientales consiste en preparar un boceto del lugar - mediante una lista de los procedimientos vigentes (descansos, normas, etc.) y de las actividades realizadas durante el día de la observación-y anotar el contexto de las conductas inapropiadas cuando éstas se dan. Inmediatamente antes y después de las observaciones de los niños y del personal, se pueden llevar a cabo observaciones del ambiente, mediante lis- tas de control, de cinco minutos de duración.

Los maestros o los miembros del personal deberían turnarse en la obtención de datos sobre los niños, el personal y el entorno. Situarse fuera del sistema para observar la interacción y las variables ambientales, casi siempre es útil para comprender y rediseñar el marco educativo.

\section{CUBRIR LAS NECESIDADES ESPECIALES DE NIÑOS FISICAMENTE DISMINUIDOS}

Los niños físicamente disminuidos presentan necesidades especiales que pueden cubrirse estructurando el aula de forma selectiva. Dado que casi no hay investigaciones sobre diseños ambientales para niños impedidos de preescolar, se propone la lista que viene a continuación como medio para determinar si un marco específico cubre las necesidades de determinados niños. Algunas de las cuestiones de la lista también pueden servir para evaluar escenarios para niños sin impedimentos físicos.

- ¿Cómo se le presenta el entorno al niño? ¿Hay cosas interesantes para ver y mirar, como ventanas, espejos, acuarios y juguetes?

- ¿Hay espacio suficiente para que los niños en silla de ruedas o de movimientos torpes puedan moverse libremente de un lugar a otro?

- ¿Hay estanterías y mesas a un nivel confortable para la altura de los niños? ¿Hay en cada área de actividad un sitio (preferiblemente más de uno) en el que un niño pueda acomodarse?

- Las estanterías, mesas, lavabos y otras instalaciones, ¿son lo suficientemente fuertes y resistentes como para aguantar el peso de un niño con poca movilidad que necesite apoyo?

- ¿Hay dispositivos protésicos (como una argolla para ponerse en pie) de fácil acceso para que los niños puedan adquirir práctica en ponerse en pie o sentarse sin ayuda de los adultos cuando están llevando a cabo alguna actividad?

- ¿Pueden los niños con dificultades de movimiento tener acceso a algunos de los juguetes y materiales sin necesitar ayuda?

- El nivel sonoro y las condiciones acústicas de la sala, ¿son satisfactorias para un niño con deficiencias auditivas o que lleve un audífono? ¿Hay alguna zona tranquila especial para que los niños puedan trabajar con un mínimo de distracciones debidas al ruido? 
atención de niños con visión deficiente? Los contrastes de color y luz, ¿corroboran los contrastes de textura y altura?

- Las señales (colores, cambios de nivel, divisiones) que designan distintas áreas, ¿̇son claras y coherentes?

- ¿Hasta qué punto se ha diseñado el ambiente para que los niños lleven a cabo actividades autónomas? ¿Con qué frecuencia aprovechan los niños estas oportunidades? ¿Necesitan los niños algún tipo de instrucción para hacerlo?

- El diseño de la sala, ¿permite delimitar lugares tranquilos y lugares sociales que se adecúen a los diversos humores y necesidades de los niños?

El diseño de ambientes de apoyo empieza con la determinación de los comportamientos que se desean obtener de los niños en el marco correspondiente a una actividad determinada. Deberian obtenerse datos conductuales y ambientales que sirvieran de base para la toma de decisiones y para determinar los efectos de los nuevos diseños. No hay ningún diseño que pueda considerarse el mejor para todos los casos. El diseño ideal es aquel que estimula eficazmente los comportamientos que son adecuados a las capacidades y necesidades de los niños.
La investigación de aspectos especificos de la planificación ambiental para niños disminuidos es muy escasa. Debería llevarse a cabo un análisis de resultados múltiples sobre los efectos de las variables ligadas al marco educativo en el comportamiento de los niños, así como. análisis sistémicos que pongan de manifiesto la interacción entre las condiciones ambientales y el comportamiento del personal, para comprobar los beneficios de los marcos educativos centrados en el niño. 